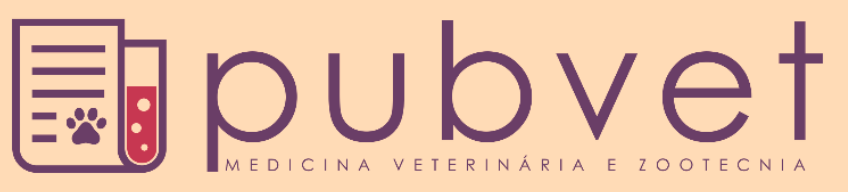

https://doi.org/10.31533/pubvet.v13n12a463.1-6

\title{
Alopecia psicogênica em gato tratada com Terapia Neural: Relato de caso
}

\author{
Bruna Aparecida Lima Gonçalves ${ }^{1 * \bullet}$, Leonardo Rocha Vianna ${ }^{2}$, Camila de Castro Andrade ${ }^{3 \bullet}$ \\ ${ }^{I}$ Médica Veterinária formada pela Pontifícia Universidade Católica de Minas Gerais Betim (PUC MINAS), Mestranda em Ciência Animal \\ pela Universidade Federal de Minas Gerais (UFMG), acupunturista, terapeuta neural. \\ ${ }^{2}$ Médico Veterinário formado na UFMG, Mestre em clínica e cirurgia pela UFMG, acupunturista, terapeuta neural, médico chinês, Ten. \\ Coronel médico veterinário reformado da PMMG e especialista em medicina natural. \\ ${ }^{3}$ Médica veterinária formada pela Pontifícia Universidade Católica de Minas Gerais (PUC MINAS), residência no Centro de Estudos em \\ clínica e cirurgia de animais (CECCA) da Pontifícia Universidade Católica de Minas Gerais Betim \\ *Autor para correspondência.E-mail: bruna_alg@yahoo.com.br
}

Resumo. A Terapia Neural (TN) é uma forma de tratamento que consiste na utilização de anestésicos locais em baixas concentrações em diversas áreas corporais do paciente, escolhidas pelo histórico de vida dele. As bases científicas se iniciaram com os russos Pavlov e Speransky posteriormente com os irmãos alemães Huneke, também tem importância Pischinger e Hans H. Reckeweg dentre outros. É praticada na Áustria, Alemanha, Argentina, Brasil, Canada, Chile, Colômbia, Costa Rica, Cuba, Espanha, Estados Unidos, México, Suíça, Turquia e alguns outros países. Consiste em uma terapia reguladora que busca ativar os mecanismos autorreguladores do organismo. O objetivo deste trabalho foi demonstrar a efetividade da Terapia Neural em um animal com quadro clínico de alopecia psicogênica.

Palavras chaves: anestésicos locais, alopecia psicogênica felina, terapia neural

\section{Psychogenic Alopecia in a Cat Treated by Neural Therapy: Case Report}

\begin{abstract}
Neural Terapy (NT) is a form of treatment that consists in the use of local anesthetics in low concentrations in diverse body areas of the patien, chosen by his life history. The scientific bases have begun with the russians Pavlov and Speransky, later with the german brothers Huneke, Pischinger and Hans H. Reckeweg have importance too, among others. It is practiced in Austria, Germany, Argentina, Brazil, Canada, Chile, Colombia, Costa Rica, Cuba, Spain, United States, Mexico, Switzerland, Turkey and some other countries. It consists in a regulatory therapy that seeks to activate the organism selfregulating mechanisms. The objective of this work was to demonstrate the effectiveness of Neural Therapy in an animal with psychogenic alopecia.
\end{abstract}

Keywords: feline psychogenic alopecia, local anesthetics, neural therapy

\section{Alopecia psicógena en un gato tratado con Terapia Neural: reporte de caso}

Resumen. La Terapia Neural es una forma de tratamiento que consiste en el uso de anestésicos locales en bajas concentraciones en diversas áreas del cuerpo del paciente, elegidos por su historia de vida. La base científica comenzó con los rusos Pavlov y Speransky más tarde con los hermanos alemanes Huneke, también tiene importancia Pischinger y Hans H. Reckeweg entre otros. Se practica en Austria, Alemania, Argentina, Brasil, Canadá, Chile, Colombia, Costa Rica, Cuba, España, Estados Unidos, México, Suiza, Turquía y algunos otros países. Consiste en una terapia reguladora que busca activar 
los mecanismos autorreguladores del organismo. El objetivo de este estudio fue demostrar la efectividad de la Terapia Neural en un animal con cuadro clínico de alopecia psicógena.

Palabras claves: alopecia psicógena felina, anestésicos locales, terapia neural

\section{Introdução}

A ansiedade é um importante causa de problemas comportamentais em gatos e pode se traduzir de diversas formas, como micção, vocalizações inapropriadas e grooming (comportamento de autolimpeza) excessivo (Ghaffari \& Sabzevari, 2010; Seksel \& Lindeman, 1988). Dermatite ou alopecia psicogênica é a condição caracterizada pelo grooming intenso e prolongada, lambedura e/ou mordidas, gerando alopecia e lesões cutâneas, na qual outras causas orgânicas foram descartadas (Sawyer et al., 1999; Talamonti et al., 2017; Virga, 2003).

Dermatites e alopecias de origem comportamental são raras em gatos. Geralmente as lesões são bilaterais e simétricas e qualquer região do corpo que o animal possa lamber pode ser acometida. São áreas comuns, a região ventral do abdome, partes internas das coxas, faces mediais dos membros posteriores (Hnilica \& Patterson, 2017). Com a cronicidade, as lesões alopécicas podem apresentar espessamento e hiperpigmentação (Barbosa et al., 2013). Raças orientais, como por exemplo, o Siamês e o Birmanês, são mais susceptíveis devido ao seu temperamento mais nervoso (Sawyer et al., 1999). A enfermidade é fruto da ansiedade do animal, que por sua vez pode ser resultado de diversos fatores, como mudanças no ambiente ou de domicílio, introdução de um novo animal ou bebê em casa, separação do tutor, hospitalização, competição e hierarquia dentro do lar (Miller et al., 2013).

Durante a consulta podem ser observados e palpados os pêlos quebrados na região acometida, e normalmente as áreas repilam com facilidade com o uso do colar elizabetano. É comum observar grande quantidade de pelos nas fezes dos gatos (Virga, 2003). O diagnóstico da dermatite psicogênica é essencialmente por exclusão, requerendo uma abordagem multidisciplinar, geral, dermatológica, neurológica e laboratorial (Talamonti et al., 2017). Os principais diagnósticos diferenciais a serem descartados compreendem doenças que cursam com prurido, tais como as dermatopatias alérgicas (alimentar e atopia), infecções fúngicas, presença de ectoparasitas e demodiciose (Ghaffari \& Sabzevari, 2010; Miller et al., 2013).

Pfeiffer et al. (1999) recomendam o tratamento medicamentoso apenas para os gatos refratários ao tratamento conservador ou em caso de automutilação, devido aos efeitos colaterais dos fármacos utilizados. Estes autores relataram um caso de retenção urinária em um felino com o uso da clomipramina, um antidepressivo tricíclico amplamente utilizado nesta afecção (Ghaffari \& Sabzevari, 2010). A medicação geralmente é instituída por 30 a 60 dias e outras opções terapêuticas incluem buspirona, amitriptilina, naloxona, haloperidol, diazepam e fenobarbital (Hnilica \& Patterson, 2017). O uso da fluoxetina também é relatado com sucesso. A modificação ambiental e a remoção dos fatores estressantes para o animal devem ser feitas quando possível, em combinação à medicação, sendo essenciais para a resolução da dermatite psicogênica (Hnilica \& Patterson, 2017; Miller et al., 2013; Sawyer et al., 1999).

A Acupuntura e a Terapia Neural são medicinas complementares regulatórias minimamente invasivas e praticamente desprovidas de efeitos colaterais que estão relacionadas entre si e seguem uma linha holística (Weinschenk, 2012).

A Terapia Neural é um sistema médico complexo que lida com o paciente como um todo, acredita na interação entre mente, corpo, emoções e ambiente, e isso tem impacto sobre os processos fisiológicos e patológicos do organismo, que são coordenados pelo sistema nervoso, ou seja, um estímulo irritativo em um determinado local do corpo o afetará como um todo (Toscano \& Pinilla, 2012). Pode-se chamar de processos irritativos as enfermidades antigas, cicatrizes, traumas, entre outros (Faber, 1989).

Teve início em 1905 com a descoberta da procaína por Alfred Eihon e as primeiras pesquisas de Spiess e em 1906, iniciou a terapia dos anestésicos locais que se desenvolveu na Alemanha e antiga URSS, foi então batizada como Terapia Neural. Foi proposta inicialmente pelos irmãos alemães médicos Walter e Ferdinand Huneke em 1925 e envolve a aplicação de pequenas quantidades de anestésicos locais específicos e diluídos, em várias áreas corporais onde se suspeita de alterações ou campos 
interferentes como, por exemplo, em cicatrizes, pontos dolorosos, pontos de acupuntura, dermátomos para espinhais, nervos periféricos entre outros (Castro, 2011; Molano et al., 2014).

Os processos patológicos podem ser denominados como campos interferentes, os quais devem ser encontrados e são tratados através da aplicação de anestésicos locais, como cloridrato de procaína, lidocaína, em concentrações que variam de $0,35 \%$ a $1,0 \%$, cujas propriedades elétricas e químicas permitem respostas corporais locais e distantes (Gonçalves et al., 2019b; Vianna \& Gonçalves, 2017).

Pischinger em meados do século XX observou normalização da resistência elétrica após aplicação de anestésicos locais em cicatrizes que antes apresentavam resistências elétricas elevadas, e desaparecimento dos sintomas clínicos imediato em alguns casos (Castro, 2011; Egli et al., 2015; Faber, 1989).

A procaína possui potencial de 290 milivolts, causando repolarização da célula e retornando ao seu potencial de membrana equilibrado (Burrel, 2008). As vias de aplicação podem ser no local dolorido, na pele, pontos gatilhos, musculatura, inserções de ligamentos, periósteo, tendões, cápsulas articulares, articulações, nervos periféricos etc. A ação é direta pela melhora da circulação local e efeito anti-inflamatório. Sabe se que $70 \%$ dos pontos de acupuntura são pontos gatilhos e todos os pontos de acupuntura podem ser estimulados pela TN (Molano et al., 2014; Vianna \& Gonçalves, 2016, 2017). Pode ser também aplicada no segmento, em uma área de projeção dos sintomas como raízes dos nervos, gânglios vegetativos, zonas de Head, ou em um campo interferente que é um "distúrbio crônico" em qualquer área do corpo capaz de produzir alterações locais ou à distância. Como por exemplo: uma cicatriz, amigdalite crônica, dentes inclusos, corpo estranho, cistos, restos de raízes de dentes, fraturas, patologias obstétricas, calos ósseos etc (Egli et al., 2015; Fischer, 2006; Koval, 2014). A Terapia Neural estimula o sistema nervoso por meio de estímulos específicos no corpo, permitindo que ocorra um autorregulação, com o objetivo de combater a doença (Burrel, 2008; Castro, 2011). Leva em consideração a individualidade por meio da história de vida do paciente (Gonçalves et al., 2019a; Gonçalves et al., 2019b; Toscano \& Pinilla, 2012). Segundo BravoMonsalvo et al. (2008) a dose máxima do cloridrato de procaína a 0,7\% quando utilizada de forma intradermal é de sete $\mathrm{mg} / \mathrm{kg}$, em um estudo em cães com dermatite atópica utilizou se também a dose endovenosa de $0,1 \mathrm{mg} / \mathrm{kg}$.

O objetivo deste trabalho é relatar o caso de um felino com alopecia psicogênica tratado com sucesso, sem medicações convencionais, exclusivamente pela Terapia Neural.

\section{Relato de caso}

Felino macho, castrado, dois anos de idade, foi atendido no Centro de Estudos em Clínica e Cirurgia em Animais (CECCA - PUC Minas) com queixa de perda de pelo em locais onde apresentava também prurido, que começou há cerca de oito meses antes da consulta. Histórico de tratamento tópico e oral com itraconazol e griseofulvina, com resposta insatisfatória. Tratava-se de um animal que não tinha contato com a rua, e nem com outros animais. Havia crianças dentro de casa e durante as ausências da tutora o gato se coçava e se lambia com mais intensidade. A alimentação, à base de ração seca, estava normal, assim como a ingestão hídrica.

Ao exame físico foram constatadas lesões alopécicas secas nos flancos e na parte interna da coxa e tarso esquerdos (Figura 1). Também havia lesões pequenas no membro posterior direito, cabeça e região dorsal. Demais parâmetros clínicos normais. Como exames complementares foram solicitados tricograma, raspado de pele e cultura para fungos. No tricograma foram notadas diversas pontas fraturadas por esforço, e bulbos em equilíbrio anágem/telógem. O raspado de pele foi negativo para ácaros e fungos, bem como a cultura que também foi negativa.

Ao primeiro retorno, estavam presentes novas lesões em tórax e na cauda. $\mathrm{O}$ felino então foi encaminhado para atendimento especializado em Terapia Neural, devido à suspeita de uma alteração comportamental, uma vez que causas orgânicas haviam sido descartadas.

Ao total do tratamento foram três sessões de Terapia Neural com intervalos de 21 dias, as aplicações foram de cloridrato de procaína a $0,7 \%$ nos acupontos VG16, VG20, B44 e na cicatriz da orquiectomia, sendo essa somente na primeira sessão. No último retorno, o animal estava calmo e as lesões repilaram completamente (Figura 2). 


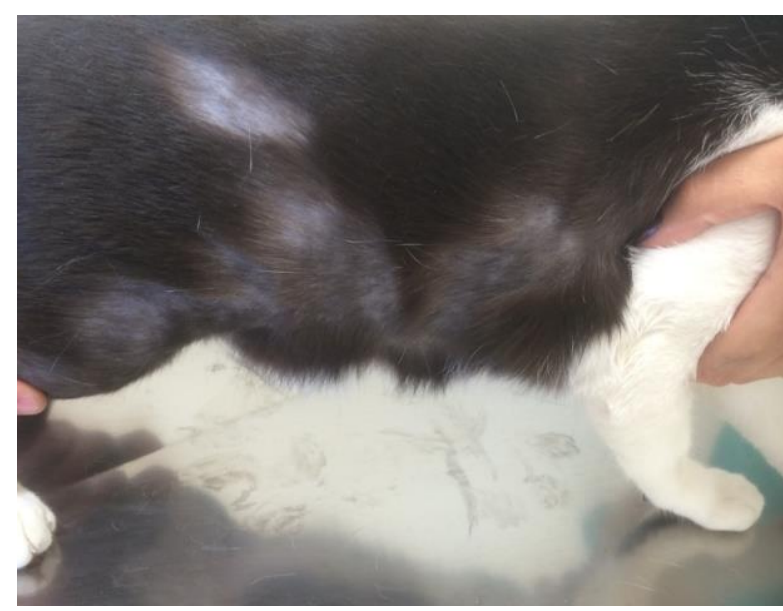

Figura 1. Gato Apresentando lesões alopécicas secas nos flancos e na parte interna da coxa e tarso

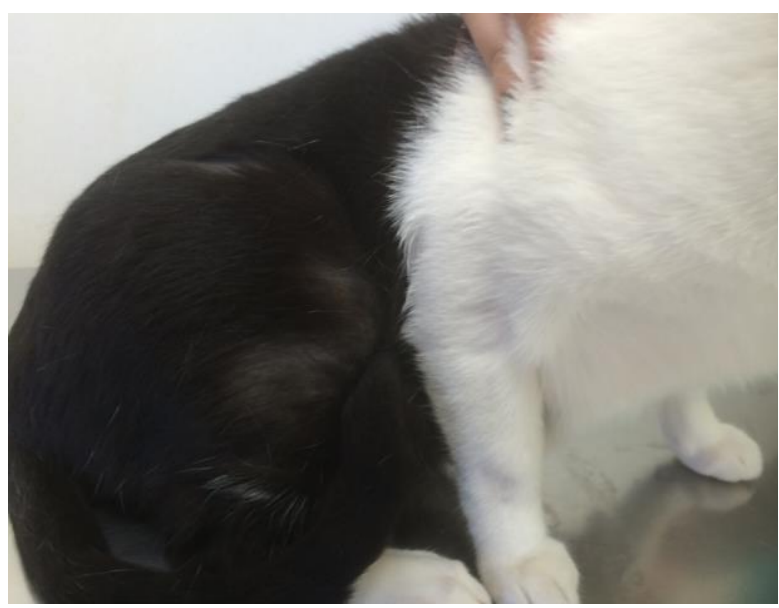

Fifura 2. Gato apresentando as lesões completamente repiladas após tres sesoes de terapia neuronal

\section{Discussão}

Os sinais clínicos apresentados pelo paciente, juntamente ao histórico, permitiram o diagnóstico, por exclusão, de alopecia psicogênica (Talamonti et al., 2017). Causas orgânicas, como a presença de ectoparasitas, dermatites alérgicas e a presença de fungos (Miller et al., 2013) foram descartadas a partir do exame físico e complementares. A distribuição das lesões está em acordo com o descrito por Hnilica \& Patterson (2017).

A presença de crianças em casa e separação da tutora foram os possíveis fatores estressantes encontrados na anamnese (Miller et al., 2013), gerando ansiedade e que por sua vez se traduziu em grooming excessivo. Este comportamento exacerbado produz quebra na estrutura dos pelos (Ghaffari \& Sabzevari, 2010), fato que foi observado no tricograma. Como se tratava de fatores ambientais não mutáveis, o foco do tratamento foi o felino. Neste caso, a Terapia Neural, que consistiu na aplicação de pequenas quantidades de solução de procaína a $0,7 \%$ em pontos de acupuntura (acupontos) foi preconizada em vez da terapia alopática. Os acupontos são regiões específicas da pele com propriedades elétricas únicas e intimamente relacionadas às terminações nervosas, nervos, vasos sanguíneos, feixes musculares, mastócitos, periósteos, entre outras estruturas, pelas quais é possível o acesso ao sistema nervoso central (Scognamillo-Szabó \& Bechara, 2001). O estímulo nos acupontos gera reações locais que se propagam até o encéfalo. Neste local são liberadas substâncias como as endorfinas, encefalinas e dimorfinas, as quais possuem efeito analgésico. Além disso, participam deste processo as vias descendentes serotoninérgicas e encefalinérgicas, que podem participar da liberação de substâncias opioides sistemicamente (Nozabieli et al., 2000).

A característica do ponto de acupuntura associado à Terapia Neural poderia explicar a eficácia do método de tratamento escolhido, uma vez que a patogenia da dermatite psicogênica pode estar diretamente atrelada à produção de endorfinas mediante o comportamento estereotipado de lambedura em resposta à situação de estresse, sendo que estas substâncias diminuem o comportamento de autolimpeza (Barbosa et al., 2013). Este também é o efeito esperado com o uso dos antidepressivos, classe de fármacos utilizados nesta enfermidade, os quais atuam como ansiolíticos e diminuem a recaptação de serotonina e noraeprinefina no sistema nervoso central, entretanto acompanhados por graus variáveis de efeitos colaterais (Pfeiffer et al., 1999), o que não acontece com a Terapia Neural.

\section{Considerações finais}

A alopecia psicogênica pode ser muito desafiadora para o clínico de pequenos animais, uma vez que consiste em um diagnóstico por exclusão. Através deste relato de caso foi comprovada a eficácia da Terapia Neural, sendo possível alcançar a cura clínica de um processo patológico de origem comportamental sem o uso de alopatia alguma, e por consequência, sem efeitos colaterais. 


\section{Referências bibliográficas}

Barbosa, A. S., Del Nero, B. \& Ambrósio, C. E. (2013). Terapia homeopática em dermatopatias de gatos-revisão de literatura. Acta Veterinaria Brasilica, 7(1):29-37.

Bravo-Monsalvo, A., Vázquez-Chagoyán, J., Gutiérrez, L. \& Sumano, H. (2008). Clinical efficacy of neural therapy for the treatment of atopic dermatitis in dogs. Acta Veterinaria Hungarica, 56(4):459469.

Burrel, S. E. (2008). Salud, enfermaria y terapia neural.

Castro, R. A. (2011). Bases para la terapia neural em caninos y felinos. Buenos Aires, Argentina: Dunken.

Egli, S., Pfister, M., Ludin, S. M., de la Vega, K. P., Busato, A. \& Fischer, L. (2015). Long-term results of therapeutic local anesthesia (neural therapy) in 280 referred refractory chronic pain patients. $B M C$ complementary and alternative medicine, 15(200):1-9.

Faber, W. J. (1989). A Review of neural therapy - Rapid therapeutic response for nerve and fascial lesions. Journal of Orthomolecular Medicine, 4(3):174-177.

Fischer, L. (2006). Terapia neural según Huneke fundamentos técnicas, aplicación práctica. México: Hippokrates.

Ghaffari, M. S. \& Sabzevari, A. (2010). Successful management of psychogenic alopecia with buspirone in a crossbreed cat. Comparative Clinical Pathology, 19(3):317-319.

Gonçalves, B. A. L., Vianna, L. R. \& Andrade, C. C. (2019a). Terapia Neural no tratamento do megaesôfago congênito em cão - relato de caso. PUBVET, 13(9):1-6.

Gonçalves, B. A. L., Vianna, L. R., Fernandes, A. L., Teixeira, A. C. B. \& Amaral, K. P. (2019b). Tratamento com Terapia Neural em cão com sequela de cinomose: Relato de caso. PUBVET, 13(7):1-6.

Hnilica, K. A. \& Patterson, A. P. (2017). Autoimmune and immune-mediated skin disorders. In K. A. Hnilica \& P. A.P. (Eds.), Small animal dermatology: a color atlas and therapeutic guide. Saint Louis, USA: Elsevier.

Koval, P. R. (2014). Medicina para o ser singular com dor persistente ou outros problemas complexos. Buenos Aires, Argentina: Ediciones incertidumbre.

Miller, W. H., Griffin, C. E., Campbell, K. L. \& Muller, G. H. (2013). Muller and Kirk's Small Animal Dermatology. Philadelphia, USA: Elsevier Health Sciences.

Molano, M. L. B., Bonilla, L. B. P., Dussan, E. H. B. \& Londoño, C. A. V. (2014). Anatomo-functional correlation between head zones and acupuncture channels and points: a comparative analysis from the perspective of neural therapy. Evidence-Based Complementary and Alternative Medicine, 2014118.

Nozabieli, A. J. L., Fregonesi, C. E. P. T. \& Fregonesi, D. A. (2000). Correlação dos canais de acupuntura com a neuroanatomia e a neurofisiologia. Arquivos de Ciências da Saúde da UNIPAR, 4(3):263-268.

Pfeiffer, E., Guy, N. \& Cribb, A. (1999). Clomipramine-induced urinary retention in a cat. The Canadian Veterinary Journal, 40(4):265.

Sawyer, L. S., Moon-Fanelli, A. A. \& Dodman, N. H. (1999). Psychogenic alopecia in cats: 11 cases (1993-1996). Journal of the American Veterinary Medical Association, 21471-74.

Scognamillo-Szabó, M. V. R. \& Bechara, G. H. (2001). Acupuntura: bases científicas e aplicações. Ciência Rural, 31(6):1091-1099.

Seksel, K. \& Lindeman, M. J. (1988). Use of clomipramine in the treatment of anxiety-related and obsessive-compulsive disorders in cats. Australian Veterinary Journal, 76(5):317-321.

Talamonti, Z., Cannas, S. \& Palestrini, C. A. (2017). Case of tail self-mutilation in a cat. Macedonian Veterinary Review, 40(1):103-107.

Toscano, T. F. \& Pinilla, B. L. B. (2012). Los principios de la terapia neural desde los fundamentos del nervismo hasta la neurociencia actua. Revista de La Universidade Industrial de Santander, 4457-65. 
Vianna, L. R. \& Gonçalves, B. A. L. (2016). Nasce uma nova especialidade na Medicina Veterinaria Brasileira: Terapia Neural e Odontologia NeuroFocal. Revista Veterinária e Zootecnia, 13016-21.

Vianna, L. R. \& Gonçalves, B. A. L. (2017). Entenda a terapia neural. Revista CFMV, 7444-47.

Virga, V. (2003). Behavioral dermatology. The Veterinary Clinics of North America. Small Animal Practice, 33(2):231-251.

Weinschenk, S. (2012). Neural therapy - a review of the therapeutic use of local anesthetics. Acupuncture and Related Therapies, 1(1): 5-9., 1(1):5-9.

Recebido: 30 de outubro, 2019.

Aprovado: 28 de novembro, 2019.

Publicado: 6 de janeiro, 2020.

Licenciamento: Este artigo é publicado na modalidade Acesso Aberto sob a licença Creative Commons Atribuição 4.0 (CC-BY 4.0), a qual permite uso irrestrito, distribuição, reprodução em qualquer meio, desde que o autor e a fonte sejam devidamente creditados. 